\title{
NOUVELLE
}

\section{Ingénierie tissulaire du parenchyme rénal}

\section{Une hypothèse thérapeutique?}

François Jouret, Yves Pirson
Service de Néphrologie, Cliniques Universitaires Saint-Luc, Université Catholique de Louvain, avenue Hippocrate 10, 1200 Bruxelles, Belgique. yves.pirson@uclouvain.be
Une question d'actualité, comment pallier la détérioration de la fonction rénale?

Les reins sont responsables de l'épuration quotidienne de notre sang et participent ainsi activement à l'homéostasie de tout notre organisme. La détérioration irréversible, aiguë ou chronique, de la fonction rénale nécessite un traitement de substitution, intermittent ou continu, afin de prévenir l'accumulation mortelle de l'eau et des métabolites toxiques. Les modalités de ce traitement sont, ou bien la greffe d'un nouveau rein, ou bien l'épuration extracorporelle par l'hémodialyse ou la dialyse péritonéale. Le nombre insuffisant d'organes disponibles limite le recours à la transplantation rénale, tandis que les complications, notamment infectieuses et cardio-vasculaires, de la dialyse péjorent le pronostic vital des patients traités par ces techniques. Si l'on y ajoute l'incidence croissante de l'insuffisance rénale terminale, en majeure partie liée au vieillissement de la population et à l'épidémie de diabète de type 2 observés dans les pays industrialisés, on comprend l'intérêt grandissant suscité par la recherche de méthodes alternatives de remplacement rénal.

À côté des études visant à améliorer les résultats de la transplantation et de la dialyse [14], des approches innovantes d'ingénierie cellulaire et tissulaire se sont récemment développées. On peut les classer en quatre catégories selon le principe de base [1]. Le « rein artificiel » consiste en un filtre issu de la nanotechnologie implantable sur la circulation sanguine et susceptible d'être alimenté en cellules rénales humaines [2]. La «rénovation rénale » utilise les potentialités des cellules souches dérivées de la moelle osseuse et/ou du rein lui-même afin de réparer in situ l'organe endommagé [3]. Le « rein en culture » consiste à développer in vitro et implanter in vivo un ensemble de néphrons capables d'épurer le sang et de former l'urine. Cette méthodologie nécessite soit l'initiation in vitro de la néphrogenèse [4], soit la différenciation en cellules rénales de cellules souches pluripotentes autologues (transfert nucléaire, reprogrammation de cellules somatiques) [5]. Enfin, la « cooptation rénale» propose de confier aux autres organes ou à la membrane péritonéale ce rôle d'élimination des toxiques et autres déchets métaboliques. La transfection cellulaire in situ et/ou l'implantation de cellules rénales permettraient en effet le développement extra-rénal des fonctions indispensables de transport de solutés et de synthèse hormonale [6].

\section{Genèse de néphrons complets in vitro} à partir d'ébauches embryonnaires Pleines de promesses, ces quatre stratégies d'ingénierie cellulaire et tissulaire proposent des approches potentiellement complémentaires de remplacement et de maintien de la fonction rénale chez le patient insuffisant rénal. Toutes se heurtent cependant aujourd'hui à différents obstacles méthodologiques, physiopathologiques et éthiques. Leur mise au point nécessite une étroite collaboration entre ingénieurs, embryologistes et néphrologues. Les travaux récents de Rosines et al. sont un exemple encourageant d'ingénierie tissulaire de «culture » de parenchyme rénal de rat [7]. Cette méthode reproduit en fait, in vitro, les étapes principales de la néphrogenèse, conduisant, à partir d'un canal de Wolff isolé et de mésenchyme métanéphrique, à la formation d'un tissu de type rénal. Les auteurs en apportent la preuve sur les plans morphologique et fonctionnel. Bien plus, ils montrent que l'implantation in vivo de ces ébauches néphroniques sous la capsule rénale d'un rat entraîne une angiogenèse précoce et l'apparition de structures glomérulaires.

Le développement embryonnaire du rein chez les mammifères est caractérisé par trois stades successifs: le pronéphros, le mésonéphros et le métanéphros [8]. La néphrogenèse commence au moment où le bourgeon urétéral issu du canal de Wolff mésodermique pénètre le mésenchyme métanéphrique (Figure 1). L'organogenèse se poursuit par inductions répétées et réciproques entre le bourgeon urétéral et le mésenchyme métanéphrique pendant que se développe la vascularisation rénale. Le bourgeon urétéral se divise par dichotomie au sein du mésenchyme et forme progressivement le système excréteur. Parallèlement, le blastème métanéphrique se condense aux extrémités du bourgeon urétéral et se différencie en néphron via différents stades de transformation mésenchymoépithéliale. La portion distale du néphron mature fusionne avec le canal collecteur issu du bourgeon urétéral et forme ainsi 
une unité fonctionnelle continue. La néphrogenèse résulte donc d'une vague centrifuge de différenciation partant du bassinet et se dirigeant vers le cortex rénal. Récemment, les gènes activés durant la néphrogenèse du rat ont été classés en cinq groupes selon le moment de leur expression durant l'organogenèse [9]. Rappelons cependant que la transcription d'un gène ne constitue que la première étape de la différenciation cellulaire et tissulaire. $\varepsilon$ n effet, la régulation de la traduction protéique et de la machinerie post-traductionnelle par des modifications comme la glycosylation, la phosphorylation et les cascades d'activation/inhibition, est indispensable au fonctionnement cellulaire, et partant, à l'organogenèse [10].
Le défi technologique de Rosines et al. a consisté dans un premier temps à déterminer les conditions de culture suffisantes au développement et à la différenciation in vitro d'un canal de Wolff préalablement isolé de son stroma mésodermique. Techniquement, le bourgeonnement du canal de Wolff nécessitait des conditions de culture tridimensionnelles enrichies en collagène de type IV (Matrigel) et un milieu enrichi en facteurs de croissance spécifiques tels que le GDNF (glial cell-derived neutrophic factor) et le FGF (fibroblast growth factor) de type 1 et 7 . Premiers résultats intéressants, la morphologie du bourgeon urétéral ainsi induit reproduisait bien la forme en $T$ observée naturellement, et chacune des deux parties de ce T préservait isolément

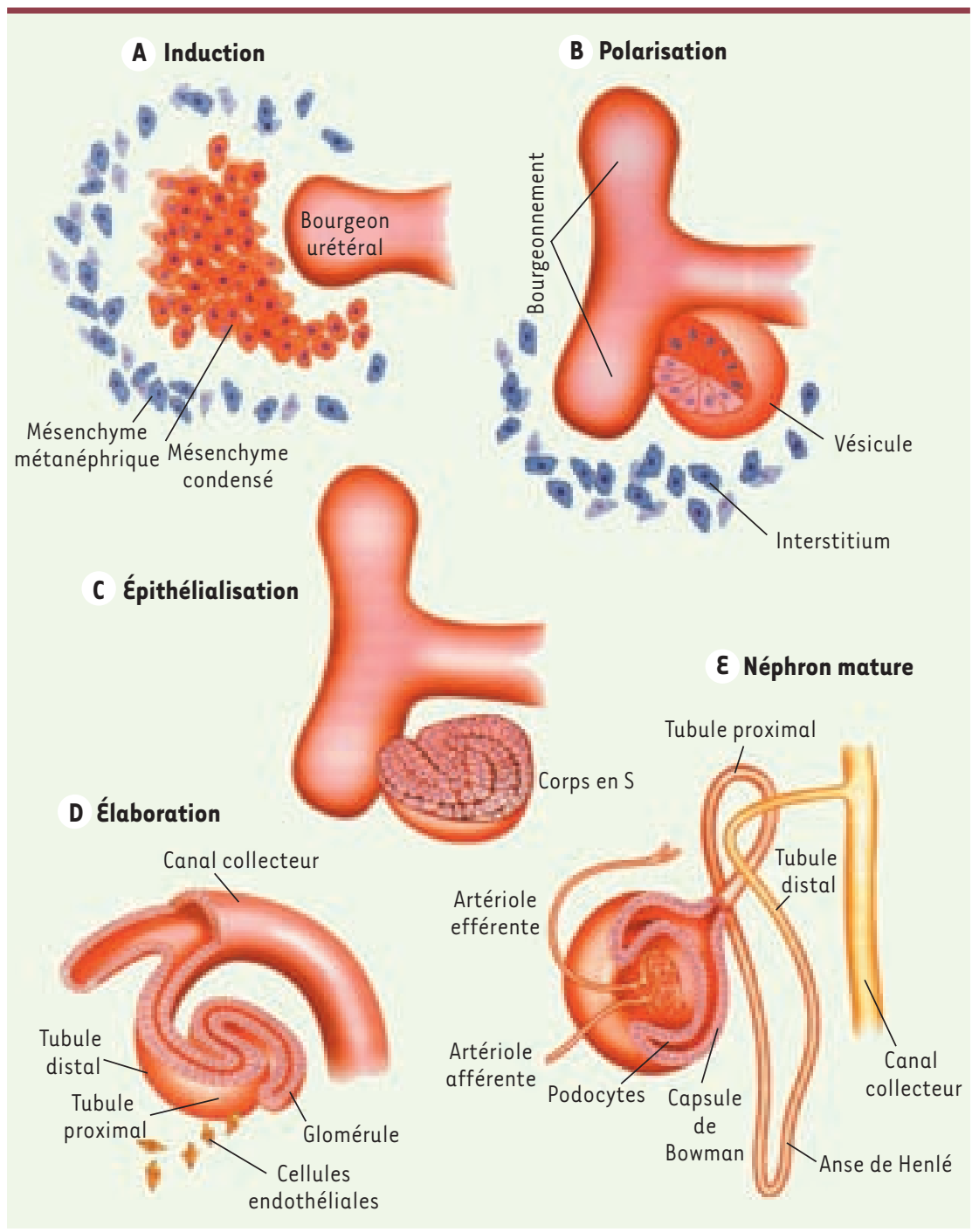

sa capacité à bourgeonner. Ce bourgeonnement dichotomique successif nécessitait un conditionnement supplémentaire du milieu de culture par les cellules BSN du mésenchyme métanéphrique, dont la nature exacte reste à ce jour inconnue [11]. L'addition de composants de la membrane basale autres que le collagène de type IV n'accélérait pas la différenciation du canal de Wolff.

Dans un second temps, Rosines et al. ont montré qu'un bourgeon urétéral développé in vitro préservait ses capacités à induire une transformation mésenchymo-épithéliale au contact d'un mésenchyme métanéphrique fraîchement isolé d'embryons de rat. Pour la première fois, la tubulogenèse et l'établissement d'une continuité entre les deux structures au niveau des canaux collecteurs aboutissaient à la formation, in vitro, de néphrons complets [12]. En outre, le transcriptome de ces structures néphroniques était comparable à celui du rein embryonnaire de rat en fin de gestation [15], et les investigations fonctionnelles préliminaires montraient que les segments tubulaires proximaux étaient dotés d'une activité de transport apical d'anions organiques. Bien plus, ce tissu rénal créé in vitro développait rapidement une néo-vascularisation dès son implantation sous la capsule rénale d'un rat, et montrait des signes de glomérulo-

Figure 1. Morphogenèse d'un néphron. A. La pénétration du bourgeon urétéral au sein $d u$ mésenchyme métanéphrique induit l'agrégation des cellules mésenchymateuses en regard de ses extrémités. B-C. L'agrégat cellulaire s'organise en vésicule rénale. Le bourgeon urétéral se divise de façon dichotomique avec induction successive du mésenchyme métanéphrique. Le stroma situé autour du bourgeon urétéral renferme les cellules souches. $\boldsymbol{D}-\boldsymbol{\varepsilon}$. À partir du stade de «corps en $S$ », la vésicule rénale s'allonge. Son extrémité proximale est envahie par les cellules endothéliales primitives et se différencie en glomérule, tandis que sa portion distale forme les différentes parties du tubule rénal et fusionne avec le canal collecteur provenant du bourgeon urétéral (adapté de [8]). 
genèse caractérisée par l'expression d'un marqueur endothélial, PECAM-1 (platelet endothelial cell adhesion molecule, ou CD31). La fonctionnalité de ces structures glomérulaires reste cependant inconnue.

\section{Perspectives :}

reconstruction néphronique à partir de cellules souches embryonnaires?

L'ensemble de ces observations démontre pour la première fois que la manipulation in vitro des structures mésodermiques et métanéphriques permet, dans certaines conditions, d'obtenir un tissu doté de caractéristiques morphologiques et fonctionnelles proches de celles d'un néphron. Étant donné que chaque bourgeon du canal de Wolff préservait sa capacité d'induction réciproque, plusieurs ébauches néphroniques pourraient être obtenues à partir d'une quantité restreinte de matériel embryonnaire. La limitation principale de cette approche réside néanmoins dans la nécessité d'isoler les structures rénales primitives riches en cellules souches pluripotentes à partir d'un embryon. Reproduire l'organogenèse rénale in vitro, à partir de cellules souches embryonnaires, amniotiques ou adultes permettrait de contourner cette limitation technique et éthique. En outre, la modification génétique de ces cellules, notamment par transfection, pourrait améliorer leur immunocompatibilité voire leur fonctionnalité. Partant, de nouvelles stratégies de xénogreffe cellulaire pourraient se développer à partir de cellules embryonnaires réputées moins immunogènes [13].

\section{En conclusion}

La caractérisation fine des différentes étapes de la néphrogenèse et leur reproduction fiable en conditions in vitro représentent une stratégie nouvelle et prometteuse de remplacement et de maintien de la fonction rénale chez le patient insuffisant rénal. $\diamond$ Engineered rat kidney tissue

\section{RÉFÉRENCES}

1. Braam B, Verhaar MC, Blankestijn P, et al. Technology insight: Innovative options for end-stage renal disease: from kidney refurbishment to artificial kidney. Nat Clin Pract Nephrol 2007 ; 3 : 564-72.

2. Humes HD, Fissell WH, Tiranathanagul K. The future of hemodialysis membranes. Kidney Int 2006; 69 : 1115-9.

3. Little MH. Regrow or repair: potential regenerative therapies for the kidney. J Am Soc Nephrol 2006; $17: 2390-401$
4. Steer DL, Bush KT, Meyer TN, et al. A strategy for in vitro propagation of rat nephrons. Kidney Int 2002 ; 62 : 1958-65.

5. Lanza RP, Chung HY, Yoo JJ, et al. Generation of histocompatible tissues using nuclear transplantation. Nat Biotechnol $2002 ; 20$ : 689-96.

6. Henderson LW. Future developments in the treatment of end-stage renal disease: a North American perspective. Am J Kidney Dis 2000 ; 35 : S106-16.

7. Rosines $\varepsilon$, Sampogna RV, Johkura K, et al. Staged in vitro reconstitution and implantation of engineered rat kidney tissue. Proc Natl Acad Sci USA 2007 ; $104: 20938-43$.

8. Bard J. The metanephros. In : Vize PD, Woolf AS, Bard JBL, eds. The kidney, from normal development to congenital disease. Orlando: Academic Press (Elsevier), 2003 : 139-43.

9. Stuart RO, Bush KT, Nigam SK. Changes in global gene expression patterns during development and maturation of the rat kidney. Proc Natl Acad Sci USA $2001 ; 98: 5649-54$.

10. Baum M, Quigley R, Satlin L. Maturational changes in renal tubular transport. Curr Opin Nephrol Hypertens $2003 ; 12$ : 521-6.

11. Qiao J, Sakurai H, Nigam SK. Branching morphogenesis independent of mesenchymal-epithelial contact in the developing kidney. Proc Natl Acad Sci USA 1999 ; $96: 7330-5$.

12. Rogers SA, Lowell JA, Hammerman NA, et al. Transplantation of developing metanephroi into adult rats. Kidney Int $1998 ; 54: 27-37$.

13. Dekel B, Burakova T, Arditti FD, et al. Human and porcine early kidney precursors as a new source for transplantation. Nat Med $2003 ; 9: 53-60$.

14. Pessione F, Cantrelle C, Savoye $\varepsilon$, et al. Activité de prélèvement et de greffe d'organes en France en 2006 Med Sci (Paris) 2007 ; 23 : 761-4.

15. De Rouffignac C. Analyse de l'expression des gènes dans le néphron de l'homme. Med Sci (Paris) 2004 ; $20: 480-3$.

\title{
Congrès Franco-Israélien
}

\author{
Regulatory $\mathbf{T}$ cells, tolerance and immunotherapy
}

\author{
14-15 Septembre 2008 \\ Mishkenot Sha'ananim, Jerusalem, Israël
}

\section{À LA MÉMOIRE DE KETTY SCHWARTZ}

\section{Comité d'organisation}

David Klatzmann (Président), Sonia Berrih Aknin (Vice-Présidente), Ronen Alon, Irun Cohen, Odile Cohen-Haguenauer, Martine Guigon-Enriquez et Miry Souroujon

\author{
Renseignements et inscriptions \\ Martine.guigon@wanadoo.fr
}

